\title{
A DEGENERATE MONGE-AMPÈRE EQUATION AND THE BOUNDARY CLASSES OF KÄHLER CONES
}

\author{
Damin Wu, Shing-Tung Yau, and Fangyang Zheng
}

\section{Introduction}

It is well known that a numerically effective (nef) line bundle on a compact complex manifold $M^{n}$ may not admit any smooth Hermitian metric whose curvature is everywhere nonnegative. The first such example were discovered by Demailly, Peternell, and Schneider in 1994 [3]. They showed that for a non-splitting extension

$$
0 \rightarrow \mathcal{O} \rightarrow \mathcal{E} \rightarrow \mathcal{O} \rightarrow 0
$$

on an elliptic curve $C$, the line bundle $L$ dual to the tautological line bundle of the projectivized bundle $M^{2}=\mathbf{P}(\mathcal{E})$ does not admit any smooth Hermitian metric with nonnegative curvature. In fact, they showed that any singular Hermitian metric on $L$ with nonnegative curvature must have logarithmic singularity, so the metric cannot even be continuous. Clearly, $L$ is a nef line bundle on the ruled surface $M$, since $\mathcal{E}$ is nef on $C$.

Here we should point out that, dated back to 1973, the second author in fact gave a talk on this example in the AMS conference at Stanford. He thought that this would give a counterexample to the Calabi conjecture. The mistake was found a few months later. The mistake was due to different definitions of semipositivity in this example.

In this article, we are interested in knowing under what conditions, any nef line bundles, or more generally any boundary classes of the Kähler cone, will always be representable by a smooth (or $C^{1,1}$ ) closed $(1,1)$ form that is everywhere nonnegative. The existence of such forms often has direct geometric applications in the study of Monge-Ampère foliations or Kähler submersions.

The existence problem can often be interpreted as a degenerate Monge-Ampère equation, which in general would be difficult to solve. Here as an exploration and a test case, we prove the existence of smooth nonnegative $(1,1)$ forms for any boundary class of the Kähler cone when the manifold satisfies certain curvature conditions.

Theorem 1. Let $\left(M^{n}, g\right)$ be a compact Kähler manifold satisfying the following curvature condition: for any orthonormal tangent frame $\left\{e_{1}, \ldots, e_{n}\right\}$ at any $x \in M$, and

Received by the editors July 10, 2008. 
for any real numbers $a_{1}, \ldots, a_{n}$ :

$$
\sum_{i, j=1}^{n} R_{i \bar{i} \bar{j}}\left(a_{i}-a_{j}\right)^{2} \geq 0
$$

Then any boundary class of the Kähler cone of $M^{n}$ can be represented by a $C^{\infty}$ closed $(1,1)$ form that is everywhere nonnegative.

Note that when $n=2$, the condition $(*)$ simply means $R_{u \bar{u} v \bar{v}} \geq 0$ for any pair of orthogonal tangent vectors $u, v$ of type $(1,0)$, namely, $M^{2}$ has nonnegative orthogonal bisectional curvature. This is certainly a very restrictive condition, and it is possible that any such manifold actually admit Kähler metric with nonnegative bisectional curvature, thus being one of the spaces in Mok's solution to the generalized Frankel's conjecture [6].

For $n>2$, the condition $(*)$ becomes much less restrictive than nonnegative orthogonal bisectional curvature. It would be an interesting question to try to classify all manifolds satisfying the curvature condition $(*)$, as a generalization to Mok's Theorem.

The referee of the article kindly pointed out to us the following fact: The above curvature condition $(*)$ appeared implicitly for the first time in the literature in 1965, in the work [1] by Bishop and Goldberg. In that paper, the authors showed that any compact Kähler manifold $M^{n}$ with positive bisectional curvature must have its second Betti number equal to 1.

It would be interesting to know what kind of restriction the second Betti number must obey under the curvature condition $(*)$, say under the strict inequality case (namely, the left hand side is positive whenever $a_{i}$ are not all equal). Notice that the product of $\mathbf{C P}^{1}$ (equipped with a sufficiently positively curved metric) with another curve always satisfies the curvature condition $(*)$ in the strict sense. So we cannot expect the second Betti number to always be 1 , even when $n=2$. On the other hand, as proved in [5] (see the proof in front of Lemma 1), on a compact Kähler manifold $M^{n}$ satisfying the curvature condition $(*)$, any harmonic $(1,1)$ form must be parallel. So in particular, the Hodge number $h^{1,1}(M)$ is equal to 1 if $M$ is locally irreducible.

Note that as a consequence of Theorem 1, we know that the ruled surface $M^{2}$ in the aforementioned example of the second author and Demailly-Peternell-Schneider does not admit any Kähler metric with nonnegative orthogonal bisectional curvature. On the other hand, $M^{2}$ clearly has nef tangent bundle.

\section{A degenerate Monge-Ampère Equation}

Let $\left(M^{n}, g_{0}\right)$ be a compact Kähler manifold. Denote by

$$
H(M)=H_{\mathbf{R}}^{1,1}(M)=H^{1,1}(M) \cap H^{2}(M, \mathbf{R})
$$

the vector space of real $(1,1)$ classes. Write $\mathcal{K}(M)$ for the Kähler cone in $H(M)$, namely, the convex cone formed by all the cohomology classes that can be represented 
by smooth closed $(1,1)$ forms that are everywhere positive. We are interested in the boundary set $\mathcal{B}=\overline{\mathcal{K}} \backslash \mathcal{K}$ of the Kähler cone. We will call a (non-trivial) cohomology class $\alpha$ in $\mathcal{B}$ a boundary Kähler class or simply a boundary class of $M$. We want to know when $\alpha$ can be represented by a closed, smooth $(1,1)$ form that is everywhere nonnegative.

As one could imagine, the case $\alpha^{n}>0$ and the case $\alpha^{n}=0$ behaves quite differently. The former means the class $\alpha$ is 'nef' and 'big' in the algebraic geometry terminology, and is in general easier to deal with. The latter case means $\alpha$ is nef but not big, which is a more degenerate situation, corresponding to the volume collapsing case under differential geometric terms. In this article we will deal with a special situation in this latter case.

Let $\omega_{0}$ be the Kähler form of $g_{0}$. Consider a path in $H(M)$ from $\left[\omega_{0}\right]$ to $\alpha$ :

$$
\alpha_{t}:=(1-t)\left[\omega_{0}\right]+t \alpha, \quad t \in[0,1] .
$$

Note that for $0 \leq t<1, \alpha_{t}$ lies in the Kähler cone. Then

$$
a(t):=\frac{1}{V} \int_{M} \alpha_{t}^{n}
$$

is positive for $0 \leq t<1$, and $a(1)=0$. Here we denote by

$$
V=\int_{M} \omega_{0}^{n} .
$$

Let us fix a smooth $(1,1)$-form $\eta$ in the class $\alpha$ (certainly if $\eta$ happens to be nonnegative then we are done.). Since $a(t) \omega_{0}^{n}$ defines a smooth volume form on $M$, by a theorem of the second author [7], there exists a smooth function $u_{t}$ on $M$, unique up to a constant, satisfying the following equations

$$
\begin{aligned}
\left(\omega_{0}+t\left(\eta-\omega_{0}\right)+d d^{c} u_{t}\right)^{n} & =a(t) \omega_{0}^{n}, \\
\omega_{0}+t\left(\eta-\omega_{0}\right)+d d^{c} u_{t} & >0,
\end{aligned}
$$

for all $0 \leq t<1$. If there is a smooth limit of $u_{t}$, say $u_{*}$, as $t \rightarrow 1$, then $\eta+d d^{c} u_{*}$ will be a desired nonnegative $(1,1)$ form representing the nef class $\alpha$.

From now on, let us consider a slightly more general case: Let $\omega_{0}$ be a Kähler form on $M$. Let $\Phi$ be a $d$-closed $(1,1)$ form on $M$, such that the cohomology class represented by $\omega_{0}+t \Phi$ is positive for each $0 \leq t<1$. In other words, for each $0 \leq t<1$, there exists a smooth function $f_{t}$ on $M$ such that

$$
\omega_{0}+t \Phi+d d^{c} f_{t}>0 \quad \text { on } M .
$$

We assume that

$$
\int_{M}\left(\omega_{0}+\Phi\right)^{n}=0
$$

Our goal is to find a smooth solution $v$ satisfying equations

$$
\begin{aligned}
\left(\omega_{0}+\Phi+d d^{c} v\right)^{n} & =0, \\
\omega_{0}+\Phi+d d^{c} v & \geq 0 .
\end{aligned}
$$

We remark that (2.2) is exactly the compatibility condition for (2.3). 
Theorem 2. Let $\omega_{0}$ and $\Phi$ be given as above. Suppose that the curvature tensor of $\omega_{0}$ satisfies the condition $(*)$ in Theorem 1 . Then there exists a smooth solution $v$ for (2.3) and (2.4).

In particular, let $\Phi=\eta-\omega_{0}$ in $(2.1)$, we obtain a desired nonnegative smooth $(1,1)-$ form $\eta+d d^{c} h$ which represents the boundary class $\alpha$. This will establish Theorem 1 for the case of a $(1,1)$-class $\alpha$ on the boundary of the Kähler cone such that $\alpha^{n}=0$. But the same proof works of course when $\alpha^{n}>0$ in exactly the same way, so Theorem 1 follows.

Our approach for Theorem 2 is by the perturbation method. Let

$$
\gamma(t)=\frac{1}{V} \int_{M}\left(\omega_{0}+t \Phi\right)^{n}, \quad \text { for all } t \in \mathbb{R} .
$$

Then $\gamma(t)$ is a smooth function which is positive on $[0,1)$; and (2.2) is equivalent to $\gamma(1)=0$. Again, for each $0 \leq t<1$, by the second author's theorem we have a solution $v_{t}$, unique up to a constant, satisfying

$$
\begin{aligned}
\left(\omega_{0}+t \Phi+d d^{c} v_{t}\right)^{n} & =\gamma(t) \omega_{0}^{n} \\
\omega_{0}+t \Phi+d d^{c} v_{t} & >0 .
\end{aligned}
$$

To solve (2.3) and (2.4), it suffices to show that there is a smooth limit for a subsequence of $\left\{v_{t}\right\}$ as $t \rightarrow 1$.

Let us normalize the solution by setting

$$
\int_{M} v_{t} \omega_{0}^{n}=0
$$

for each $0 \leq t<1$. We claim that the unique solution $v_{t}$ of (2.5), (2.6), and (2.7) depends smoothly on $t$ for $0 \leq t<1$. This will be proved in the next section. The argument is to apply the implicit function theorem, similar to the proof of the openness in the continuity method (see [7]).

Then we can differentiate the equation (2.5) with respect to $t$. It turns out that the function $v_{t}-t \dot{v}_{t}$ would be important in the a priori estimates. Here we denote by $\dot{a}=\partial a / \partial t$. In particular, we obtain that

$$
\Delta^{\prime}\left(v_{t}-t \dot{v}_{t}\right)=C(t)-S .
$$

Here $\Delta^{\prime}$ is the Laplacian for $\omega_{t}=\omega_{0}+t \Phi+d d^{c} v_{t}, C(t)$ is a constant depending on $t$, and $S$ is the trace of $\omega_{0}$ with respect to $\omega_{t}$. On the other hand, motivated by the second author's generalized Schwarz Lemma ([8]), one can estimate $S$ via the Chern-Lu formula ([2] and [4]). Then the curvature condition $(*)$ and the maximum principle will enable us to conclude that $S$ depends only on $t$. Thus, by (2.8) and solving an ordinary differential equation, we obtain a smooth solution for $t=1$. This finishes the proof of Theorem 2 . 


\section{A smooth family of solutions}

Let $v_{t}$ be the unique smooth solution satisfying

$$
\begin{aligned}
\left(\omega_{0}+t \Phi+d d^{c} v_{t}\right)^{n} & =\gamma(t) \omega_{0}^{n}, \\
\omega_{0}+t \Phi+d d^{c} v_{t} & >0, \\
\int_{M} v_{t} \omega_{0}^{n} & =0,
\end{aligned}
$$

for each $0 \leq t<1$. We would like to show that $v_{t}$ depends smoothly on $t$. To see this, we will use the implicit function theorem for Banach spaces. We denote by

$$
\mathcal{E}^{k, \alpha}=\left\{h \in C^{k, \alpha}(M) ; \int_{M} h \omega_{0}^{n}=0 .\right\}
$$

for each positive integer $k$ and $\alpha \in(0,1)$. Then each $\mathcal{E}^{k, \alpha}$ is itself a Banach space, as a closed subspace in the Hölder space $C^{k, \alpha}(M)$. Let us fix a $k$ and an $\alpha$. We define

$$
\mathcal{M}(t, u)=\frac{\left(\omega_{0}+t \Phi+d d^{c} u\right)^{n}}{\gamma(t) \omega_{0}^{n}}-1,
$$

for all $u \in C^{k+2, \alpha}(M)$ and $0 \leq t<1$. Then $\mathcal{M}(t, u)$ defines a smooth map from $[0,1) \times \mathcal{E}^{k+2, \alpha}$ to $\mathcal{E}^{k, \alpha}$.

Now for an arbitrary $t \in[0,1)$ with $v_{t}$ satisfying (3.1), (3.2), and (3.3), we have $v_{t} \in \mathcal{E}^{k+2, \alpha}$ and

$$
\mathcal{M}\left(t, v_{t}\right)=0 .
$$

We need to show that $D_{2} \mathcal{M}\left(t, v_{t}\right)$, the partial Fréchet derivative of $\mathcal{M}$ at $\left(t, v_{t}\right)$ with respect to $v_{t}$, is a linear isomorphism from $\mathcal{E}^{k+2, \alpha}$ to $\mathcal{E}^{k, \alpha}$. Here we identify a Banach space with its the tangent space at a point. Observe that, for any $h \in \mathcal{E}^{k+2, \alpha}$,

$$
\begin{aligned}
D_{2} \mathcal{M}\left(t, v_{t}\right)(h) & =\left.\frac{d}{d s} \mathcal{M}\left(t, v_{t}+s h\right)\right|_{s=0} \\
& =\frac{n\left(\omega_{0}+t \Phi+d d^{c} v_{t}\right)^{n-1} \wedge d d^{c} h}{\gamma(t) \omega_{0}^{n}} \\
& =\Delta^{\prime} h .
\end{aligned}
$$

Here $\Delta^{\prime}$ is the Laplacian associated with the metric

$$
\omega_{t}=\omega_{0}+t \Phi+d d^{c} v_{t} .
$$

Note that the kernel of $\Delta^{\prime}$ consists of constant functions; so does its cokernel. Then the former implies the injectivity of $D_{2} \mathcal{M}\left(t, v_{t}\right)$ on $\mathcal{E}^{k+2, \alpha}$; while the latter, together with the Schauder theory imply the surjectivity of $D_{2} \mathcal{M}\left(t, v_{t}\right)$. Thus, $D_{2} \mathcal{M}\left(t, v_{t}\right)$ is a linear isomorphism. It then follows from the implicit function theorem that $v_{t}$ depends smoothly on $t$. 


\section{Estimates}

We denote by $S$ the trace of $\omega_{0}$ with respect to $\omega_{t}$, where

$$
\omega_{t}=\omega_{0}+t \Phi+d d^{c} v_{t}, \quad 0 \leq t<1
$$

By differentiating (3.1) with respect to $t$ we get

$$
n \omega_{t}^{n-1} \wedge\left(\Phi+d d^{c} \dot{v}_{t}\right)=\dot{\gamma} \omega_{0}^{n} .
$$

Here we denote by $\dot{a}=\partial a / \partial t$. Moreover, observe that

$$
n \omega_{t}^{n-1} \wedge\left(t \Phi+d d^{c} v_{t}\right)=n \omega_{t}^{n}-n \omega_{t}^{n-1} \wedge \omega_{0} .
$$

Combining (4.1) and (4.2) we obtain

$$
n \omega_{t}^{n-1} \wedge d d^{c}\left(v_{t}-t \dot{v}_{t}\right)=(n \gamma-t \dot{\gamma}) \omega_{0}^{n}-n \omega_{t}^{n-1} \wedge \omega_{0} .
$$

Dividing both sides by $\omega_{t}^{n}$ yields

$$
\Delta^{\prime}\left(v_{t}-t \dot{v}_{t}\right)=(n-t \dot{\gamma} / \gamma)-S .
$$

Next we will derive an estimate for $S$, motivated by the idea of the generalized Schwarz Lemma by the second author [8].

Proposition 4.1. Let $M$ be a Kähler manifold of complex dimension $n$. Let $g_{i \bar{j}}$ and $g_{i \bar{j}}^{\prime}$ be two Kähler metrics on $M$, and $R_{i \bar{j}}, R_{i \bar{j}}^{\prime}$ be, respectively, their Ricci curvature tensors. Suppose that

$$
R_{\xi \bar{\xi}}^{\prime} \geq R_{\xi \bar{\xi}}
$$

for any type $(1,0)$ tangent vector $\xi$ on $M$. We assume, in addition, that the curvature tensor of $g_{i \bar{j}}$ satisfies the condition $(*)$ in Theorem 1. That is, for any orthonormal tangent frame $\left\{e_{1}, \ldots, e_{n}\right\}$ at any $x \in M$, and for any nonnegative numbers $a_{1}, \ldots, a_{n}$, we have

$$
\sum_{i, j=1}^{n} R_{i \bar{i} \bar{j} \bar{j}}\left(a_{i}-a_{j}\right)^{2} \geq 0 .
$$

Let $S$ be the trace of $g_{i \bar{j}}$ with respect to $g_{i \bar{j}}^{\prime}$. Then

$$
\Delta^{\prime} \log S \geq 0
$$

where $\Delta^{\prime}$ is the Laplacian for $g_{i \bar{j}}^{\prime}$.

Proof of Proposition 4.1. It is equivalent to show that

$$
\Delta^{\prime} S-\frac{\left|\nabla^{\prime} S\right|^{2}}{S} \geq 0
$$


Notice that

$$
\begin{aligned}
\Delta^{\prime} S= & \sum_{k, l} g^{\prime k \bar{l}} \frac{\partial^{2}}{\partial z^{k} \partial \bar{z}^{l}}\left(\sum_{i, j} g^{\prime i \bar{j}} g_{i \bar{j}}\right) \\
= & \sum_{i, j, k, l} g^{\prime k \bar{l}} g_{i \bar{j}} \frac{\partial^{2} g^{\prime i \bar{j}}}{\partial z^{k} \partial \bar{z}^{l}}+\sum_{i, j, k, l} g^{\prime \prime \bar{l}} g^{\prime i \bar{j}} \frac{\partial^{2} g_{i \bar{j}}}{\partial z^{k} \partial \bar{z}^{l}} \\
& +\sum_{i, j, k, l} g^{\prime k \bar{l}}\left(\frac{\partial g^{\prime i \bar{j}}}{\partial z^{k}} \frac{\partial g_{i \bar{j}}}{\partial \bar{z}^{l}}+\frac{\partial g^{\prime i \bar{j}}}{\partial \bar{z}^{l}} \frac{\partial g_{i \bar{j}}}{\partial z^{k}}\right) .
\end{aligned}
$$

We have, by direct computation,

$$
\begin{aligned}
\frac{\partial g^{\prime i \bar{j}}}{\partial \bar{z}^{l}}= & -\sum_{p, q} g^{\prime i \bar{q}} g^{\prime p \bar{j}} \frac{\partial g_{p \bar{q}}^{\prime}}{\partial \bar{z}^{l}}, \\
\frac{\partial^{2} g^{\prime i \bar{j}}}{\partial z^{k} \partial \bar{z}^{l}}= & -\sum_{p, q} g^{\prime i \bar{q}} g^{\prime p \bar{j}} \frac{\partial^{2} g_{p \bar{q}}^{\prime}}{\partial z^{k} \partial \bar{z}^{l}} \\
& +\sum_{a, b, p, q}\left(g^{\prime i \bar{b}} g^{\prime a \bar{q}} g^{\prime p \bar{p}} \frac{\partial g_{a \bar{b}}^{\prime}}{\partial z^{k}} \frac{\partial g_{p \bar{q}}^{\prime}}{\partial \bar{z}^{l}}+g^{\prime i \bar{q}} g^{\prime a \bar{j}} g^{\prime p \bar{b}} \frac{\partial g_{a \bar{b}}^{\prime}}{\partial z^{k}} \frac{\partial g_{p \bar{q}}^{\prime}}{\partial \bar{z}^{l}}\right) .
\end{aligned}
$$

Observe that

$$
-\sum_{k, l} g^{\prime k \bar{l}} \frac{\partial^{2} g_{p \bar{q}}^{\prime}}{\partial z^{k} \partial \bar{z}^{l}}=R_{p \bar{q}}^{\prime}-\sum_{a, b, k, l} g^{\prime a \bar{b}} g^{\prime k \bar{l}} \frac{\partial g_{a \bar{q}}^{\prime}}{\partial z^{k}} \frac{\partial g_{p \bar{b}}^{\prime}}{\partial \bar{z}^{l}} .
$$

Thus, the first term on the right hand side of (4.5) is given by

$$
\begin{aligned}
\sum_{i, j, k, l} g^{\prime k \bar{l}} g_{i \bar{j}} \frac{\partial^{2} g^{\prime i \bar{j}}}{\partial z^{k} \partial \bar{z}^{l}}= & \sum g^{\prime i \bar{q}} g^{\prime p \bar{j}} R_{p \bar{q}}^{\prime} g_{i \bar{j}}-\sum g_{i \bar{j}} g^{\prime i \bar{q}} g^{\prime p \bar{j}} g^{\prime a \bar{b}} g^{\prime k \bar{l}} \frac{\partial g_{a \bar{q}}^{\prime}}{\partial z^{k}} \frac{\partial g_{p \bar{b}}^{\prime}}{\partial \bar{z}^{l}} \\
& +\sum\left(g^{\prime i \bar{b}} g^{\prime a \bar{q}} g^{\prime p \bar{j}}+g^{\prime i \bar{q}} g^{\prime a \bar{j}} g^{\prime p \bar{b}}\right) g_{i \bar{j}} g^{\prime k \bar{l}} \frac{\partial g_{a \bar{b}}^{\prime}}{\partial z^{k}} \frac{\partial g_{p \bar{q}}^{\prime}}{\partial \bar{z}^{l}} \\
= & \sum g^{\prime i \bar{q}} g^{\prime p \bar{j}} R_{p \bar{q}}^{\prime} g_{i \bar{j}}+\sum g^{\prime i \bar{q}} g^{\prime a \bar{j}} g^{\prime p \bar{b}} g_{i \bar{j}} g^{\prime k \bar{l}} \frac{\partial g_{a \bar{b}}^{\prime}}{\partial g_{p \bar{q}}^{\prime}} \frac{\partial \bar{z}^{l}}{\partial \bar{z}^{l}}
\end{aligned}
$$

Let us now choose a normal coordinate system $\left(z^{1}, \ldots, z^{n}\right)$ at a point of $M$ such that at the point

and

$$
g_{i \bar{j}}=\delta_{i j}, \quad \frac{\partial g_{i \bar{j}}}{\partial z^{k}}=\frac{\partial g_{i \bar{j}}}{\partial \bar{z}^{l}}=0,
$$

for $i, j, k, l=1, \ldots, n$. Then we have

$$
g_{i \bar{j}}^{\prime}=\delta_{i j} g_{i \bar{i}}^{\prime}
$$

$$
\sum_{i, j, k, l} g^{\prime k \bar{l}} g_{i \bar{j}} \frac{\partial^{2} g^{\prime i \bar{j}}}{\partial z^{k} \partial \bar{z}^{l}}=\sum_{i} \frac{R_{i \bar{i}}^{\prime}}{\left(g_{i \bar{i}}^{\prime}\right)^{2}}+\sum_{i, p, k} \frac{\left|\partial g_{i \bar{p}}^{\prime} / \partial z^{k}\right|^{2}}{\left(g_{i \bar{i}}^{\prime}\right)^{2} g_{p \bar{p}}^{\prime} g_{k \bar{k}}^{\prime}}
$$

The second term on the right of (4.5) is given by

$$
\sum_{i, j, k, l} g^{\prime k \bar{l}} g^{\prime i \bar{j}} \frac{\partial^{2} g_{i \bar{j}}}{\partial z^{k} \partial \bar{z}^{l}}=-\sum_{i, k} \frac{R_{i \bar{i} k \bar{k}}}{g_{k \bar{k}}^{\prime} g_{i \bar{i}}^{\prime}} .
$$


Therefore, we have

$$
\begin{aligned}
\Delta^{\prime}(S)= & -\sum_{i, k} \frac{R_{i \bar{i} k \bar{k}}}{g_{i \bar{i}}^{\prime} g_{k \bar{k}}^{\prime}}+\sum_{i} \frac{R_{i \bar{i}}^{\prime}}{\left(g_{i \bar{i}}^{\prime}\right)^{2}} \\
& +\sum_{i, j, k} \frac{\left|\partial g_{i \bar{j}}^{\prime} / \partial z^{k}\right|^{2}}{\left(g_{i \bar{i}}^{\prime}\right)^{2} g_{j \bar{j}}^{\prime} g_{k \bar{k}}^{\prime}} .
\end{aligned}
$$

Observe that

$$
\begin{aligned}
\left|\nabla^{\prime} S\right|^{2} & =\sum_{k, l} g^{\prime k \bar{l}} \frac{\partial S}{\partial z^{k}} \frac{\partial S}{\partial \bar{z}^{l}} \\
& =\sum_{k} \frac{1}{g_{k \bar{k}}^{\prime}}\left|\sum_{i} \frac{\partial g_{i \bar{i}}^{\prime} / \partial z^{k}}{\left(g_{i \bar{i}}^{\prime}\right)^{2}}\right|^{2} \\
& =\sum_{k} \frac{1}{g_{k \bar{k}}^{\prime}}\left|\sum_{i} \frac{\partial g_{i \bar{i}}^{\prime} / \partial z^{k}}{\left(g_{i \bar{i}}^{\prime}\right)^{3 / 2}} \frac{1}{\left(g_{i \bar{i}}^{\prime}\right)^{1 / 2}}\right|^{2} .
\end{aligned}
$$

Applying Cauchy-Schwarz inequality yields that

$$
\left|\nabla^{\prime} S\right|^{2} \leq S \sum_{i, k} \frac{\left|\partial g_{i \bar{i}}^{\prime} / \partial z^{k}\right|^{2}}{\left(g_{i \bar{i}}^{\prime}\right)^{3} g_{k \bar{k}}^{\prime}}
$$

It follows from (4.6), (4.7), and (4.4) that

$$
\begin{aligned}
\Delta^{\prime} S-\frac{\left|\nabla^{\prime} S\right|^{2}}{S} & \geq-\sum_{i, k} \frac{R_{i \bar{i} k \bar{k}}}{g_{i \bar{i}}^{\prime} g_{k \bar{k}}^{\prime}}+\sum_{i} \frac{R_{i \bar{i}}^{\prime}}{\left(g_{i \bar{i}}^{\prime}\right)^{2}} \\
& \geq \sum_{i, k} \frac{R_{i \bar{i} k \bar{k}}}{\left(g_{i \bar{i}}^{\prime}\right)^{2}}-\sum_{i, k} \frac{R_{i \bar{i} k \bar{k}}}{g_{i \bar{i}}^{\prime} g_{k \bar{k}}^{\prime}} \\
& =\frac{1}{2} \sum_{i, k} \frac{R_{i \bar{i} k \bar{k}}}{\left(g_{i \bar{i}}^{\prime}\right)^{2}}+\frac{1}{2} \sum_{i, k} \frac{R_{i \bar{i} k \bar{k}}}{\left(g_{k \bar{k}}^{\prime}\right)^{2}}-\sum_{i, k} \frac{R_{i \bar{i} k \bar{k}}}{g_{i \bar{i}}^{\prime} g_{k \bar{k}}^{\prime}} \\
& =\frac{1}{2} \sum_{i, k} R_{i \bar{i} \bar{k} \bar{k}}\left(\frac{1}{g_{i \bar{i}}^{\prime}}-\frac{1}{g_{k \bar{k}}^{\prime}}\right)^{2} .
\end{aligned}
$$

Now we apply condition $(*)$ to obtain that

$$
S \Delta^{\prime} \log S=\Delta^{\prime} S-\frac{\left|\nabla^{\prime} S\right|^{2}}{S} \geq 0 .
$$

This finishes the proof.

In our case, we actually only need $\Delta^{\prime} S \geq 0$ to conclude that $S$ is a function depending only on $t$, since $M$ is compact. Here we have assumed that the curvature of $\omega_{0}$ satisfies $(*)$. By $(3.1)$, we have

$$
R_{i \bar{j}}^{\prime}=R_{i \bar{j}}
$$

Now observe that the right hand side of (4.3) depends only on $t$. Applying the maximum principle to $v_{t}-t \dot{v}_{t}$, we know that both sides of (4.3) will have to equal 
zero. Hence, $v_{t}-t \dot{v}_{t}$ depends only on $t$. But we also have the normalization (3.3); differentiating (3.3) with respect to $t$ yields that

$$
\int_{M} \dot{v}_{t} \omega_{0}^{n}=0
$$

Putting these together, we obtain

$$
v_{t}-t \dot{v}_{t}=0 \quad \text { on } M .
$$

By solving this ordinary differential equation we conclude that

$$
v_{t}=t h,
$$

where $h$ is a function on $M$. This implies that $h \in C^{\infty}(M)$ with

$$
\omega_{0}+t \Phi+t d d^{c} h>0 \quad \text { on } M
$$

for all $0 \leq t<1$. Letting $t \rightarrow 1$, we obtain a nonnegative smooth $(1,1)$-form

$$
\omega_{1}=\omega_{0}+\Phi+d d^{c} h
$$

which satisfies that

$$
\omega_{1}^{n}=0 .
$$

This completes the proof of Theorem 2 .

\section{Acknowledgements}

The first author would like to thank B. Guan, L. Ni, M. Ramachandran, R. Schoen, V. Tosatti, P.-M. Wong, Z. Zhang for helpful discussions. We are very grateful to the referee of this article for several corrections and helpful suggestions, which improved the readability.

\section{References}

[1] R. L. Bishop and S. I. Goldberg, On the second cohomologh group of a Kaehler manifold of positive curvature, Proc. Amer. Math. Soc. 16 (1965), 119-122.

[2] S. S. Chern, On holomorphic mappings of Hermitian manifolds of the same dimension, Proc. Symp. Pure Math. 11, Amer. Math. Soc. (1968), 157-170.

[3] J.-P. Demailly, T. Peternell, and M. Schneider, Compact complex manifolds with numerically effective tangent bundles, J. Algebraic Geom. 3 (1994), no. 2, 295-345.

[4] Y.-C. Lu, Holomorphic mappings of complex manifolds, J. Diff. Geom. 2 (1968), 299-312.

[5] A. Howard, B. Smyth, and H. Wu, On compact Kḧler manifolds of nonnegative bisectional curvature, I, Acta Math. 147 (1981), 51-56.

[6] N. Mok, The uniformization theorem for compact Kähler manifolds of nonnegative holomorphic bisectional curvature, J. Differential Geom. 27 (1988), no. 2, 179-214.

[7] S.-T. Yau, On the Ricci curvature of a compact Kähler manifold and the complex MongeAmpère equation I, Comm. Pure Appl. Math., 31 (1978), 339-411.

[8] __ A general Schwarz lemma for Kähler manifolds, Amer. J. Math. 100 (1978), 197-203. 
Department of Mathematics, Ohio State University, 1179 University Drive, Newark, OH 43055

E-mail address: dwu@math.ohio-state.edu

Department of Mathematics, Harvard University, One Oxford Street, Cambridge Ma 02138

E-mail address: yau@math.harvard.edu

Department of Mathematics, Ohio State University, 231 West 18th Avenue, Columbus OH 43210

E-mail address: zheng@math.ohio-state.edu 